\title{
Bayesian Learning of Generalized Gaussian Mixture Models on Biomedical Images
}

\author{
Tarek Elguebaly and Nizar Bouguila \\ CIISE, Faculty of Engineering and Computer Science, Concordia University, \\ Montreal, Qc, Canada H3G 2W1 \\ t_elgue@encs.concordia.ca, bouguila@ciise.concordia.ca
}

\begin{abstract}
In the context of biomedical image processing and bioinformatics, an important problem is the development of accurate models for image segmentation and DNA spot detection. In this paper we propose a highly efficient unsupervised Bayesian algorithm for biomedical image segmentation and spot detection of cDNA microarray images, based on generalized Gaussian mixture models. Our work is motivated by the fact that biomedical and cDNA microarray images both contain nonGaussian characteristics, impossible to model using rigid distributions like the Gaussian. Generalized Gaussian mixture models are robust in the presence of noise and outliers and are more flexible to adapt the shape of data.
\end{abstract}

\section{Introduction}

In recent years a lot of different algorithms were developed in the aim of automatically learning to recognize complex patterns, and to make intelligent decisions based on observed data. Machine learning, a branch of artificial intelligence, offers a principled approach for developing and studying automatic techniques capable of learning models and their parameters based on training data. Recent advances in machine learning have fascinated researchers from biology/bioinformatics community because they offer promise for the development of novel supervised and unsupervised methods that can help in specifying, detecting, and diagnosing different diseases, while at the same time increasing objectivity of the decision-making process. The relation history between biology and the field of machine learning is long and complex. The flexibility of machine learning techniques is expected to improve the efficiency of discovery and understanding in the mounting volume and complexity of biological data. Machine learning techniques have been used, for instance, in [1] for microarray analysis and classification, in [2] for DNA microarray image spot detection, in [3] for biomedical image analysis, and in 4 for multiple limb motion classification.

Mixture models are one of the machine learning techniques receiving considerable attention in different applications. Mixture models are normally used to model complex datasets. In most of biomedical applications, the Gaussian density is applied for data modeling 45. However, data are generally non-Gaussian 6]. Many studies have demonstrated that the generalized Gaussian distribution

F. Schwenker and N. El Gayar (Eds.): ANNPR 2010, LNAI 5998, pp. 207 218, 2010.

(C) Springer-Verlag Berlin Heidelberg 2010 
(GGD) can be a good alternative to the Gaussian thanks to its shape flexibility which allows the modeling of a large number of non-Gaussian signals $7 / 8$. The GGD for a variable $x \in \mathbb{R}$ is defined as follows:

$$
P(x \mid \mu, \alpha, \beta)=\frac{\beta \alpha}{2 \Gamma(1 / \beta)} e^{-(\alpha|x-\mu|)^{\beta}}
$$

where $\alpha=\frac{1}{\sigma} \sqrt{\frac{\Gamma(3 / \beta)}{\Gamma(1 / \beta)}},-\infty<\mu<\infty, \beta>0$, and $\alpha>0$, and $\Gamma($.$) is the$ Gamma function given by: $\Gamma(x)=\int_{0}^{\infty} t^{x-1} e^{-t} d t, x>0 . \mu, \alpha$ and $\beta$ denote the distribution mean, the inverse scale parameter, and the shape parameter, respectively. The GGD is flexible thanks to its shape parameter $\beta$ that controls the decay rate of the density function. In other words, $\beta$ allows the GGD to take different shapes depending on the data. Fig. 1 shows us two main reasons to use GGD. First, the parameter $\beta$ controls the shape of the pdf. The larger the value, the flatter the pdf; and the smaller the value, the more picked the pdf. Second, when $\beta=2$ and $\beta=1$, the GGD is reduced to the Gaussian and Laplacian distributions, respectively. In the past few years, several approaches have been applied for GGDs parameters estimation such as moment estimation 9], entropy matching estimation [1011], and maximum likelihood estimation [1213]. It is noteworthy that these approaches consider a single distribution. Concerning finite mixture models parameters estimation, approaches can be arranged into two categories: deterministic, and Bayesian methods. In deterministic approaches, parameters are taken as fixed and unknown, and inference is founded on the likelihood of the data. In the recent past, some deterministic approaches have been proposed for the estimation of finite generalized Gaussian mixture (GGM) models parameters (see, for instance, 14 15]). Despite the fact that deterministic approaches have controlled mixture models estimation due to their small computational time, many works have demonstrated that these methods have severe problems such as convergence to local maxima, and their tendency to overfitt the data [16] especially when data are sparse or noisy. With computational tools evolution, researchers were encouraged to implement and use Bayesian MCMC methods and techniques as an alternative approach. Bayesian methods consider parameters to be random, and to follow different probability distributions (prior distributions). These distributions are used to describe our

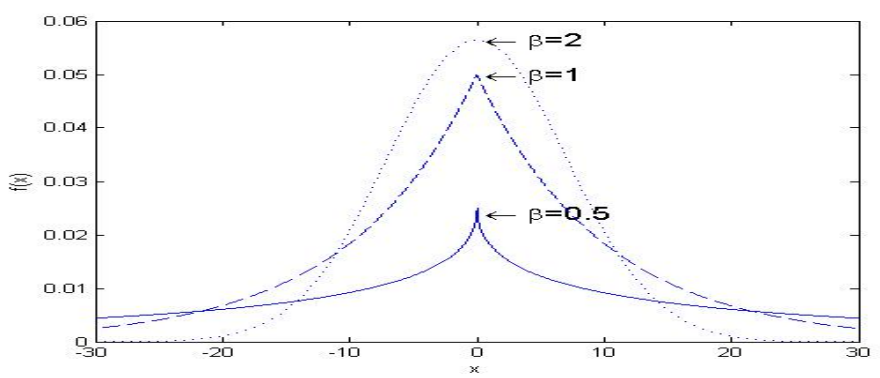

Fig. 1. Generalized Gaussian Distributions with different values of the shape parameter 
knowledge before considering the data, as for updating our prior beliefs the likelihood is used. Please refer to [16] for interesting and in depth discussions about the general Bayesian theory. In this paper, we describe a Bayesian algorithm for GGM learning and provide two examples of their applications in Biomedical/Bioinformatics fields. Biomedical image segmentation is chosen to be our first application since medical images are highly corrupted by noise, and contain non-gaussian characteristics. For the second application, we are interested in developing an algorithm capable of automatically detecting the spots in DNA microarray images.

The rest of this paper is organized as follows. Section 2 describes the Bayesian estimation algorithm. In section 3, we demonstrate the efficacy of the model on two applications. Our last section is devoted to the conclusion.

\section{Bayesian Estimation of the GGM Model}

A general Gaussian mixture with $M$ components is defined as:

$$
P(x \mid \Theta)=\sum_{j=1}^{M} P\left(x \mid \mu_{j}, \alpha_{j}, \beta_{j}\right) p_{j}
$$

where $p_{j}$ are the mixing proportions which are constrained to be non-negative and sum to one, and $p\left(x \mid \mu_{j}, \alpha_{j}, \beta_{j}\right)$ is the GGD describing component $j$. The symbol $\Theta=(\xi, p)$ refers to the whole set of parameters to be estimated, knowing that $\xi=\left(\mu_{1}, \alpha_{1}, \beta_{1}, \ldots, \mu_{M}, \alpha_{M}, \beta_{M}\right)$, and $p=\left(p_{1}, \ldots, p_{M}\right)$. The two main problems in finite mixture models are the estimation of the parameters vector $\Theta$ and the number of components $M$. Supposing that the number of classes $M$ is known then for $N$ observations , $\mathcal{X}=\left(x_{1}, \ldots, x_{N}\right)$, the likelihood corresponding to this case is:

$$
P(\mathcal{X} \mid \Theta)=\prod_{i=1}^{N} \sum_{j=1}^{M} P\left(x_{i} \mid \xi_{j}\right) p_{j}
$$

where $\xi_{j}=\left(\mu_{j}, \alpha_{j}, \beta_{j}\right)$. For each variable $x_{i}$, let $Z_{i}$, the unobserved or missing vector, be an $M$-dimensional vector that indicates to which component $x_{i}$ belongs. In other words, $Z_{i j}$ equals 1 if $x_{i}$ belongs to class $j$ and 0 , otherwise. The complete-data likelihood for this case is then:

$$
P(\mathcal{X}, Z \mid \Theta)=\prod_{i=1}^{N} \sum_{j=1}^{M}\left(P\left(x_{i} \mid \xi_{j}\right) p_{j}\right)^{Z_{i j}}
$$

where $Z=\left\{Z_{1}, Z_{2}, \ldots, Z_{N}\right\}$. Then, the estimation of each $Z_{i j}$, defined as the posterior probability that the $i$ th observation arises from the $j$ th component of the mixture is:

$$
\widehat{Z}_{i j}^{(t)}=\frac{P^{(t-1)}\left(x_{i} \mid \xi_{j}^{(t-1)}\right) p_{j}^{t-1}}{\sum_{j=1}^{M} P^{(t-1)}\left(x_{i} \mid \xi_{j}^{(t-1)}\right) p_{j}^{t-1}}
$$

where $t$ denotes the current iteration step and $\xi_{j}^{(t)}$ and $p_{j}^{(t)}$ are the current evaluations of the parameters. 
Bayesian MCMC simulation methods are applied to get the posterior distribution $\pi(\Theta \mid \mathcal{X}, Z)$. Generally MCMC methods are found on the Bayesian theory, which means that they allow for probability statements to be made directly about the unknown parameters of a mixture model, while taking into consideration prior or expert opinion. In order to get the posterior distribution using MCMC, we need to combine the prior information about the parameters, $\pi(\Theta)$, with the observed value or realization of the complete data $P(\mathcal{X}, Z \mid \Theta)$. This can be reached from Bayes formula:

$$
\pi(\Theta \mid \mathcal{X}, Z)=\frac{\pi(\Theta) P(\mathcal{X}, Z \mid \Theta)}{\int \pi(\Theta) p(\mathcal{X}, Z \mid \Theta)} \propto \pi(\Theta) P(\mathcal{X}, Z \mid \Theta)
$$

where $(\mathcal{X}, Z)$ is the complete data. With the joint distribution, $\pi(\Theta) P(\mathcal{X}, Z \mid \Theta)$, in hand we can deduce the posterior distribution (Eq. 6). Having $\pi(\Theta \mid \mathcal{X}, Z)$ we can simulate our model parameters $\Theta$, rather than computing them. Taking advantage of the missing data $Z$, we simulate $Z$ according to the posterior probability $\pi(Z \mid \Theta, \mathcal{X})$. This is done by associating with each observation $x_{i}$ a missing Multinomial variable $Z_{i} \sim \mathcal{M}\left(1 ; \widehat{Z}_{i 1} ; \ldots ; \widehat{Z}_{i M}\right)$. This choice is based on two reasons, first, we know that each $Z_{i}$ is a vector of zero-one indicator variables. Second, the probability that the $i$ th observation, $x_{i}$, arises from the $j$ th component of the mixture is given by $\widehat{Z}_{i j}$.

For $p$ simulation we need to get $\pi\left(p \mid Z^{(t)}\right)$, using Bayes rule: $\pi(p \mid Z)=\frac{\pi(Z \mid p) \pi(p)}{\int \pi(Z \mid p) \pi(p)}$ $\propto \pi(Z \mid p) \pi(p)$. This indicates that we need to determine $\pi(Z \mid p)$, and $\pi(p)$. Moreover, we know that the vector $\mathrm{P}$ is defined on the simplex $\left(0 \leq p_{j} \leq 1\right.$ and $\left.\sum_{j=1}^{M} p_{j}=1\right)$, then the typical choice, as a prior, for this vector is a Dirichlet distribution with parameters $\eta=\left(\eta_{1}, \ldots, \eta_{M}\right)$

$$
\pi(p)=\frac{\Gamma\left(\sum_{j=1}^{M} \eta_{j}\right)}{\prod_{j=1}^{M} \Gamma\left(\eta_{j}\right)} \prod_{j=1}^{M} p_{j}^{\eta_{j}-1}
$$

As for $\pi(Z \mid p)$ we have:

$$
\pi(Z \mid p)=\prod_{j=1}^{M} \pi\left(Z_{i} \mid p\right)=\prod_{i=1}^{N} \prod_{j=1}^{M} p_{j}^{Z_{i j}}=\prod_{j=1}^{M} p_{j}^{n_{j}}
$$

Where $n_{j}=\sum_{i=1}^{N} \mathbf{I}_{Z_{i j=1}}$, then we can conclude that:

$$
\begin{gathered}
\pi(p \mid Z)=\pi(Z \mid P) \pi(p)=\frac{\Gamma\left(\sum_{j=1}^{M} \eta_{j}\right)}{\prod_{j=1}^{M} \Gamma\left(\eta_{j}\right)} \prod_{j=1}^{M} p_{j}^{\eta_{j}-1} \prod_{j=1}^{M} p_{j}^{n_{j}}=\frac{\Gamma\left(\sum_{j=1}^{M} \eta_{j}\right)}{\prod_{j=1}^{M} \Gamma\left(\eta_{j}\right)} \prod_{j=1}^{M} p_{j}^{\eta_{j}+n_{j}-1} \\
\propto \mathcal{D}\left(\eta_{1}+n_{1}, \ldots, \eta_{M}+n_{M}\right)
\end{gathered}
$$

$\mathcal{D}$ denotes the Dirichlet distribution with parameters $\left(\eta_{1}+n_{1}, \ldots, \eta_{M}+n_{M}\right)$. From (Eq. 9) we can deduce that the Dirichlet distribution is a conjugate prior for the mixture proportions, which means that the prior and the posterior have 
the same form. Let us now define $\xi$ priors, which are supposed to be drawn independently. For the parameters $\xi$, we assigned independent Normal priors for the distributions means, and Gamma priors for the inverse scale and shape parameters 17 18]:

$$
\mu_{j} \sim \mathcal{N}\left(\mu_{0}, \sigma_{0}^{2}\right), \beta_{j} \sim \mathcal{G}\left(\alpha_{\beta}, \beta_{\beta}\right), \alpha_{j} \sim \mathcal{G}\left(\alpha_{\alpha}, \beta_{\alpha}\right)
$$

Where $\mathcal{N}\left(\mu_{0}, \sigma_{0}^{2}\right)$ is the normal distribution with mean $\mu_{0}$ and variance $\sigma_{0}^{2}$, $\mathcal{G}\left(\alpha_{\beta}, \beta_{\beta}\right)$ is the gamma distribution with shape parameter $\alpha_{\beta}$ and rate parameter $\beta_{\beta} \cdot \mu_{0}, \sigma_{0}^{2}, \alpha_{\beta}, \beta_{\beta}, \alpha_{\alpha}, \beta_{\alpha}$ are called the hyperparameters of the model. With this priors, we can deduce the posterior distributions for $\mu, \alpha$, and $\beta$ to be:

$$
\begin{gathered}
\pi\left(\mu_{j} \mid Z, \mathcal{X}\right) \propto \mathbf{e}^{\frac{\left(\mu_{j}-\mu_{0}\right)^{2}}{2 \sigma_{0}^{2}}+\sum_{z_{i j}=1}\left(-\alpha_{j}\left|x_{i}-\mu_{j}\right|\right)^{\beta_{j}}} \\
\pi\left(\alpha_{j} \mid Z, \mathcal{X}\right) \propto \alpha_{j}^{\alpha_{\alpha}-1} \mathbf{e}^{-\beta_{\alpha} \alpha_{j}}\left(\alpha_{j}\right)^{n_{j}} \mathbf{e}^{\sum_{z_{i j=1}}\left(-\alpha_{j}\left|x_{i}-\mu_{j}\right|\right)^{\beta_{j}}} \\
\pi\left(\beta_{j} \mid Z, \mathcal{X}\right) \propto \beta_{j}^{\alpha_{\beta-1}} \mathbf{e}^{-\beta_{\beta} \beta_{j}}\left(\frac{\beta_{j}}{\Gamma\left(1 / \beta_{j}\right)}\right)^{n_{j}} \mathbf{e}^{\sum_{z_{i j=1}}\left(-\alpha_{j}\left|x_{i}-\mu_{j}\right|\right)^{\beta_{j}}}
\end{gathered}
$$

It is quite easy to notice that we cannot simulate directly from these posterior distributions because they are not in well known forms. To solve this problem we applied the well known Metropolis-Hastings (M-H) algorithm given in [19]. The major problem in the $\mathrm{M}-\mathrm{H}$ algorithm is the choice of the proposal distribution. Random Walk M-H given in [19] is used here to solve this problem, then the proposals are considered to be: $\widetilde{\mu_{j}} \sim \mathcal{N}\left(\mu_{j}^{(t-1)}, \zeta^{2}\right), \widetilde{\alpha_{j}} \sim \mathcal{L} \mathcal{N}\left(\log \left(\alpha_{j}^{(t-1)}\right), \zeta^{2}\right)$, $\widetilde{\beta}_{j} \sim \mathcal{L} \mathcal{N}\left(\log \left(\beta_{j}^{(t-1)}\right), \zeta^{2}\right)$, where $\mathcal{L} \mathcal{N}$ is the log-normal distribution, since, we know that $\widetilde{\alpha_{j}}>0$ and $\widetilde{\beta_{j}}>0$. $\zeta^{2}$ is the scale of the random walk.

In fact, choosing a relevant model consists both of choosing its form and the number of components $M$. The integrated or marginal likelihood using the Laplace-Metropolis estimator [19] is applied in order to rate the ability of the tested models to fit the data or to determine the number of clusters $M$. The integrated likelihood is defined by 19

$$
p(\mathcal{X} \mid M)=\int \pi(\Theta \mid \mathcal{X}, M) d \Theta=\int p(\mathcal{X} \mid \Theta, M) \pi(\Theta \mid M) d \Theta
$$

where $\Theta$ is the vector of parameters of a finite mixture model, $\pi(\Theta \mid M)$ is its prior density, and $p(\mathcal{X} \mid \Theta, M)$ is the likelihood function taking into account that the number of clusters is $M$.

\section{Experimental Results}

In this section, we apply the Bayesian estimation of the GGM in biomedical image segmentation, and microarray image spot detection. We validate the algorithm by comparing it to different state of the art algorithms. In the following 
applications, we used 5000 iteration for our Metropolis-within-Gibbs sampler (we discarded the first 800 iterations as "burn-in" and kept the rest), and our specific choices for the hypeparameters were $\left(\mu_{0}, \sigma_{0}^{2}, \alpha_{\alpha}, \beta_{\alpha}, \alpha_{\beta}, \beta_{\beta}\right)=(0,1,0.2,2,0.2,2)$. To increase the sensitivity of the random walk sampler, the scale of the random walk was chosen to be $\zeta^{2}=0.01$.

\subsection{Biomedical Image Segmentation}

Image segmentation is one of the major challenges in image analysis, since image analysis tasks highly depend on how well previous segmentation is accomplished. Image segmentation is the procedure of dividing an image into different groups with each group enjoying similar properties such as texture, color, boundary, and intensity. Despite, the existence of different segmentation methods, many of them fail to provide satisfactory results when applied on biomedical images. Reasons behind this failure are numerous. First, image segmentation is strongly influenced by the quality of data and biomedical images contain different noises such as speckle, shadows which may cause the boundaries of structures to be indistinct and disconnected. Second, most of image segmentations algorithms are founded on the assumption that the data are Gaussian which is not the case for biomedical images. Further complications arise as the contrast between areas of interest in biomedical images is low, which make the extraction of the desired regions impossible as they are statistically indistinguishable. Last but not least, most of existed segmentation methods do not integrate uncertain prior knowledge.

In this section, we develop a new segmentation methodology, using the Bayesian MCMC algorithm developed in section 2. We can divide our method into two main steps: histogram adjustment, and identification of object of interest using the Bayesian GGM with the integrated likelihood. We validate our algorithm by comparing it to a state of the art segmentation algorithm [20]. This method is divided into two stages: preprocessing, and object segmentation. Preprocessing stage contains histogram adjustment, noise reduction, and layer of interest extraction using K-means algorithm. For the object segmentation a marker-controlled watershed technique is used.

The first image used is the image of a rat spleen tissue pulps (Fig. 2(a)). For visual differentiation of cellular components, the tissue section was stained with haematoxylin and eosin $(H \& E)$. Under a microscope, nuclei are usually dark blue, red blood cells orange/red, and muscle fibers deep pink/red. The feature used to differentiate red and white is the density of the lymphocytes. The white pulp has lymphocytes and macrophages surrounding central arterioles. The distribution of the lymphocytes in red pulp is much looser than those in white pulp. Evaluating the severity of infection requires identifying the white pulps. We started by transforming the color image to a gray level image (Fig. 3(a)) in order to simplify the processing procedure. For grayscale image nuclei are dark objects within a gray background. Then histogram adjustment [21] is applied on the image to increase image contrast (Fig. 33(b)). At this point, we applied our Bayesian GGM to identify the object of interest in the image (Fig. 3(c)). 


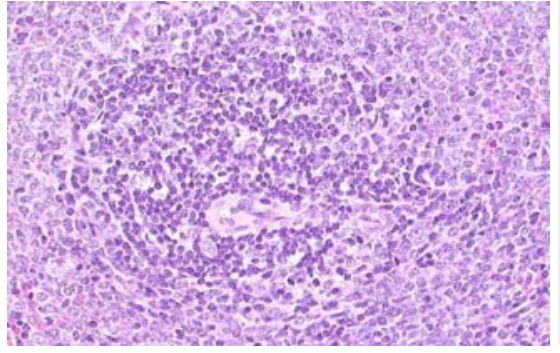

(a)

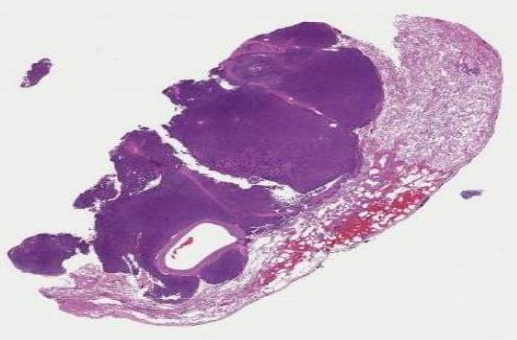

(b)

Fig. 2. Microscopic images used, (a) The rat spleen tissue pulps (Courtesy of Dr. Jinglu Tan), (b) Lung Carcinoid tumor (Courtesy of Dr. Robert Cardiff)

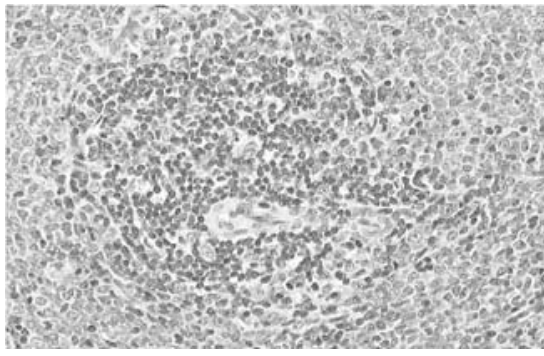

(a)

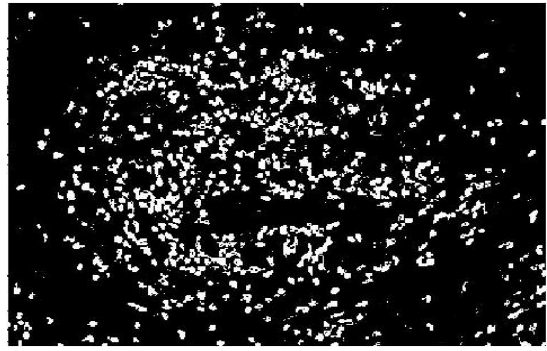

(c)

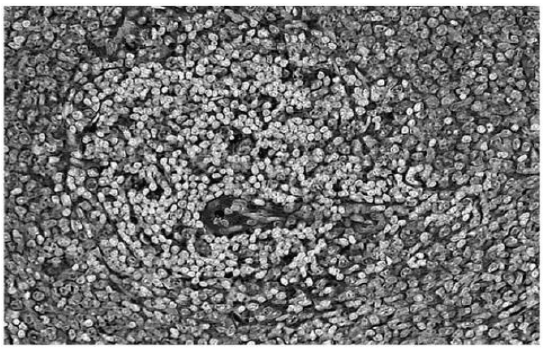

(b)

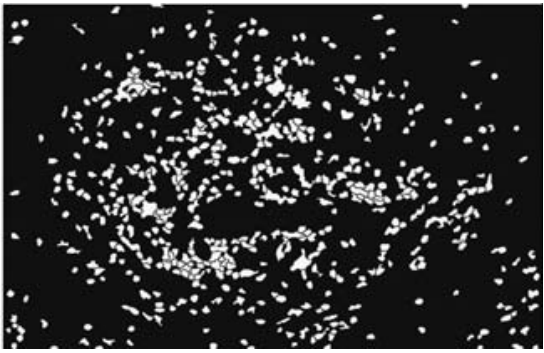

(d)

Fig. 3. The different stage outputs for the two methods on the rat spleen tissue, (a) The gray scale image, (b) The image after histogram adjustment, (c) The identified object of interest using our method, (d) The identified object of interest using the state of the art algorithm

Comparing the output from our method to the one from the watershed method (Fig. [3(d)), we can find that we were able to reach a higher identification for the infected regions. Also, the proposed method is less complex due to the fact that we did not need to use neither noise reduction, nor marker-controlled watershed techniques.

The second image is an image of a carcinoid tumor seen in the lung of eighty one years old female (Fig. 2(b)). To be able to differentiate visually the cellular 


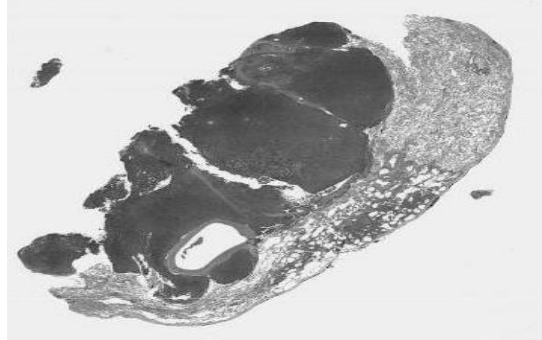

(a)

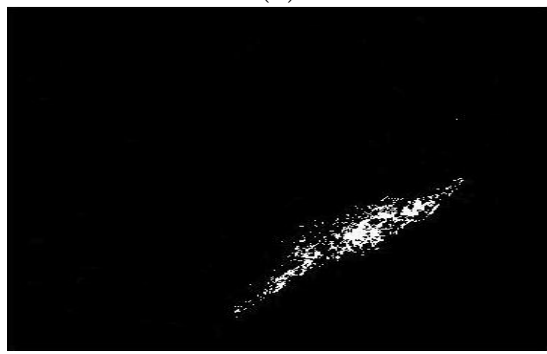

(c)

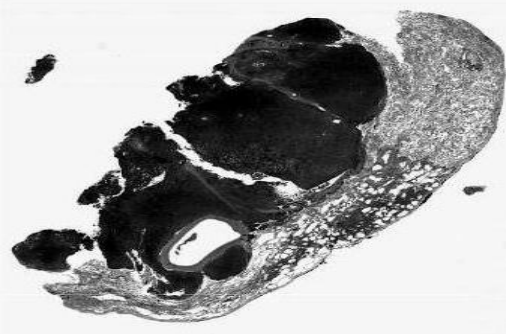

(b)

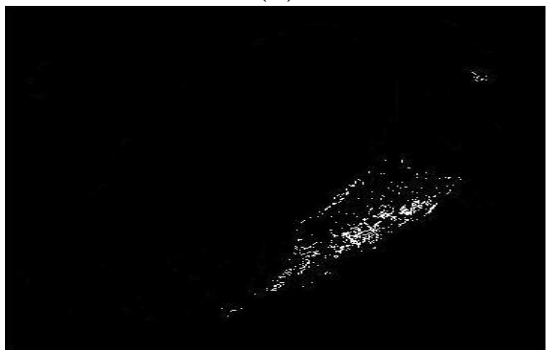

(d)

Fig. 4. The different stage outputs for the two methods on the Lung Carcinoid tumor, (a) The gray scale image, (b) The image after histogram adjustment, (c) The identified object of interest using our method, (d) The identified object of interest using the state of the art algorithm

components, the tissue section was stained with haematoxylin and eosin $(H \& E)$. The size of the tumor is of $2.5 \mathrm{~cm}$ long as shown in the image. First we transformed the image into gray scale image (Fig. 4(a)). Next, we applied the histogram adjustment on the image (Fig. 4(b)), and last we applied our algorithm on it to reach the object of interest (Fig. 4(c)). Also, it is quite clear here that our algorithm outperformed the watershed algorithm. Experimental results show that the proposed method is effective and accurate in segmenting microscopic images even without the need of noise reduction stages and marker-controlled watershed techniques to separate the touching objects.

\subsection{Spot Detection and Image Segmentation in DNA Microarray Data}

In this section, we propose an optimized clustering-based method for microarray image segmentation using GGM. Our algorithm is based on the fact that GGM is flexible to model the shape of data, and have high immunity to noise. To assess the performance of our method, we compare it to two well known algorithms: k-means clustering microarray image segmentation (SKMIS) [22], and optimized k-means microarray image (OKMIS) [23. We evaluate the segmentation performance of the three methods on the spot images from ApoA1 Data [24]. 
DNA microarray technology is a high throughput technique allowing the comprehensive measurement of the expression level of thousands of genes simultaneously in the studies of genomics for biology and medicine 25. Complementary single stranded DNA (cDNA) microarrays consist of thousands of individual DNA sequences printed in a high density array. Nowadays, microarray experiments are used to compare gene expression from two samples: target or experimental, and control. The mRNA of both biological tissues (normal and tumor) is extracted, then reversed transcribed into complementary DNA (cDNA) copy, followed by a labeling procedure using two fluorescence dyes, Cyanine Cy3 (green channel) and Cy5 (red channel). After labeling, the two samples are mixed and hybridized with the arrayed DNA sequences. Afterwards, fluorescence measurements are made for each dye separately, and the digital image scanner records the intensity level at each microarray location producing two grayscale images [26].

Image analysis is a highly important aspect of cDNA microarray experiments, as it is responsible for reducing an image of spots into a table with a measure of the intensity for each spot. Efficient, accurate and automatic analysis of cDNA spot images is necessary in order to apply this technology in different biological experiments. cDNA microarray gene expression data analysis involves three main stages: spot localization or gridding, background separation or image segmentation, and intensity estimation. Spot localization or gridding is used to identify blocks and to position rows and columns of spots within each block. Background separation or image segmentation is used to segment the image into background or foreground, and the intensity estimation step gets the red and green intensities and assigns the log ratio after background correction in order to represent the log relative abundance of each spot. These stages are quite important, since the accuracy of the resulting data is essential in posterior analysis.

In cDNA microarray experiments, noise is a challenging problem as it can be produced by laser light reflection, dust on the glass slide, and photon and electronic noise. These noises force microarray images to vary in intensity, in the spot sizes and positions. For this reason, we decided to apply the Bayesian GGM on this problem for its immunity to noise. Over the past few years, many approaches have been proposed for microarray image segmentation. Fixed circle segmentation is the first applied technique on microarray images, its idea is to assign the same circle size to all the spots. Another proposed method in order to avoid the drawback of the fixed circle segmentation is the adaptive circle segmentation technique. This algorithm fits a circle with adaptive size around each spot, in order to characterize the pixels in the circle as signal pixels and the pixels out of the circle as background pixels (i.e. foreground or background). Another technique that has been efficiently used in microarray image segmentation is clustering, since it is not restricted to a particular shape and size for the spots. Single k-means clustering microarray image segmentation (SKMIS) attempts to cluster the pixels into two groups, one for foreground, and the other for background. Therefore in SKMIS, feature vector is reduced to a single variable in the Euclidean one-dimensional space. Optimized k-means microarray image segmentation (OKMIS) not only consider the intensity of the pixel but also the shape 


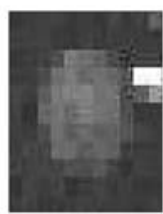

(a)

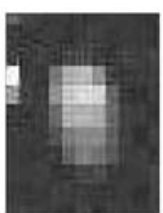

(b)

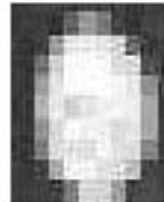

(c)

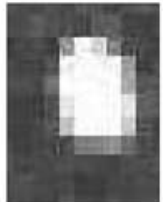

(d)

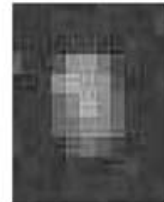

(e)

Fig. 5. Five noisy spots obtained from the $1230 \mathrm{c} 1 \mathrm{G} / \mathrm{R}$ microarray image

SKMIS
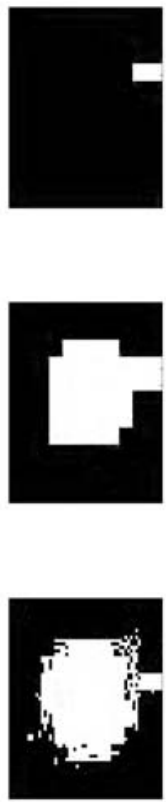

(a)
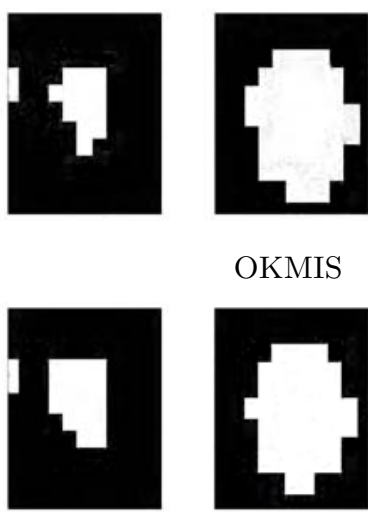

OKMIS

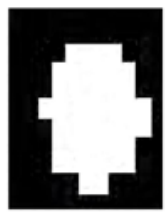

GGM

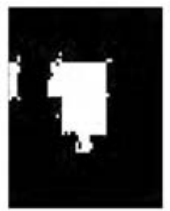

(b)

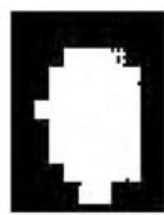

(c)
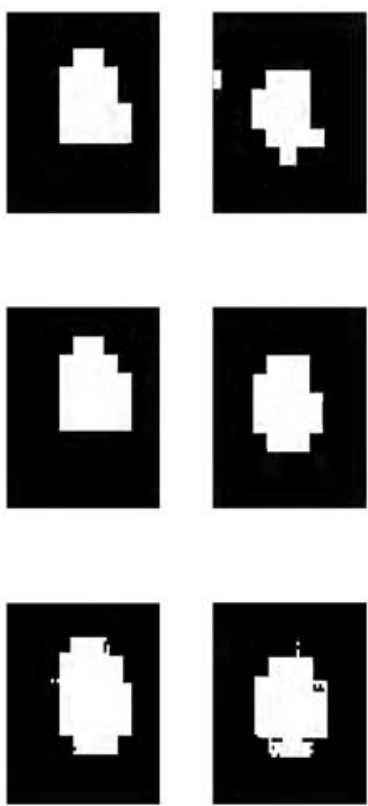

(d)

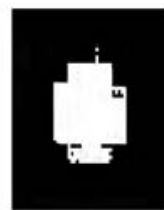

(e)

Fig. 6. The experimental results of the three methods on the five noisy spots

of the spot based on the fact that the position of the pixel could also influence the result of the clustering. Our algorithm is very simple as we only apply a two component GGM to classify the data to either foreground or background.

In order to compare GGM, OKMIS and SKMIS, we applied the three methods on the $1230 \mathrm{c} 1 \mathrm{G} / \mathrm{R}$ microarray image obtained from the ApoA1 data. Fig. 5 shows some examples for the noisy spots in our microarray image. From Fig. [ it is clear that our method was able to retrieve the true foreground from the background. We also observe that the GGM outperformed the SKMIS and OKMIS in identifying noisy pixels from foreground. Note that, the GGM was able to take the data form. Hence, the GGM is more suitable when dealing with cDNA microarray image segmentation. 


\section{Conclusion}

In this paper, we have presented a new Bayesian algorithm for biomedical image segmentation and multi-class DNA classification. Our method is based on GGM models which chief advantage is their flexibility and immunity to noise. We have used the Monte Carlo simulation technique of Gibbs sampling mixed with a Metropolis-Hasting step for parameters estimation. The Bayesian estimation of the model parameters incorporates uncertainty which disease diagnosis, for instance, are in need. For the selection of number of clusters we have used the integrated Likelihood. The experimental results show the effectiveness of the proposed method in two interesting applications.

\section{Acknowledgment}

The completion of this research was made possible thanks to the Natural Sciences and Engineering Research Council of Canada (NSERC), a NATEQ Nouveaux Chercheurs Grant, and a start-up grant from Concordia University.

\section{References}

1. Cho, S.-B., Won, H.-H.: Machine Learning in DNA Microarray Analysis for Cancer Classification. In: Proc. of the First Asia-Pacific Bioinformatics Conference, pp. 189-198 (2003)

2. Katzer, M., Kummert, F., Sagerer, G.: Methods for Automatic Microarray Image Segmentation. IEEE Transactions on NanoBioscience 2(4), 202-214 (2003)

3. Pappas, T.N.: An Adaptive Clustering Algorithm for Image Segmentation. IEEE Transactions on Signal Processing 40(4), 901-914 (1992)

4. Yonghong, H., Englehart, K.B., Hudgins, B., Chan, A.D.C.: A Gaussian Mixture Model Based Classification Scheme for Myoelectric Control of Powered Upper Limb Prostheses. IEEE Transactions on Biomedical Engineering 52(11), 1801-1811 (2005)

5. Rocke, D.M., Durbin, B.: A Model for Measurement Error for Gene Expression Arrays. Journal of Computational Biology 8(6), 557-569 (2004)

6. Bouguila, N., Ziou, D., Monga, E.: Practical Bayesian Estimation of a Finite Beta Mixture Through Gibbs Sampling and its Applications. Statistics and Computing 16(2), 215-225 (2006)

7. Gao, Z., Belzer, B., Villasenor, J.: A Comparison of the Z, E 8 , and Leech Lattices for Quantization of Low Shape-Parameter Generalized Gaussian Sources. IEEE Signal Processing Letters 2(10), 197-199 (1995)

8. Meignen, S., Meignen, H.: On the Modeling of Small Sample Distributions with Generalized Gaussian Density in a Maximum Likelihood Framework. IEEE Transactions on Image Processing 15(6), 1647-1652 (2006)

9. Sharifi, K., Leon-Garcia, A.: Estimation of Shape Parameter for Generalized Gaussian Distributions in Subband Decomposition of Video. IEEE Transactions on Circuits and Systems for Video Technology 5(1), 52-56 (1995)

10. Aiazzi, B., Alpaone, L., Baronti, S.: Estimation Based on Entropy Matching for Generalized Gaussian PDF Modeling. IEEE Signal Processing Letters 6(6), 138 140 (1999) 
11. Kokkinakis, K., Nandi, A.K.: Exponent Parameter Estimation for Generalized Gaussian Probability Density Functions with Application to Speech Modeling. Signal Processing 85(9), 1852-1858 (2005)

12. Varanasi, M.K., Aazhang, B.: Parametric Generalized Gaussian Density Estimation. The Journal of the Acoustical Society of America 86(4), 1404-1415 (1989)

13. Pi, M.: Improve Maximum Likelihood Estimation for Subband GGD Parameters. Pattern Recognition Letters 27(14), 1710-1713 (2006)

14. Allili, M.S., Bouguila, N., Ziou, D.: Finite General Gaussian Mixture Modeling and Application to Image and Video Foreground Segmentation. Journal of Electronic Imaging 17(1), 1-13 (2008)

15. Fan, S.-K.S., Lin, Y.: A Fast Estimation Method for the Generalized Gaussian Mixture Distribution on Complex Images. Computer Vision and Image Understanding 113(7), 839-853 (2009)

16. Robert, C.P.: The Bayesian Choice From Decision-Theoretic Foundations to Computational Implementation, 2nd edn. Springer, Heidelberg (2007)

17. Robert, C.P., Casella, G.: Monte Carlo Statistical Methods, 2nd edn. Springer, Heidelberg (2004)

18. Gentle, J.E., Härdle, W.: Handbook of Computational Statistics. In: Concepts and Fundamentals, vol. 1, Springer, Heidelberg (2004)

19. Lewis, S.M., Raftery, A.E.: Estimating Bayes Factors via Posterior Simulation with the Laplace-Metropolis Estimator. Journal of the American Statistical Association 90, 648-655 (1997)

20. Yu, J., Tan, J.: Object Density-Based Image Segmentation and its Applications in Biomedical Image Analysis. Computer Methods and Programs in Biomedicine 96(3), 193-204 (2009)

21. Larson, G.W., Rushmeier, H., Piatko, C.: A Visibility Matching Tone Reproduction Operator for High Dynamic Range Scenes. IEEE Transactions on Visualization and Computer Graphics 3(4), 291-306 (1997)

22. Wu, S., Yan, H.: Microarray Image Processing Based on Clustering and Morphological Analysis. In: Proc. of the First Asia Pacific Bioinformatics Conference, pp. 111-118 (2003)

23. Rueda, L., Qin, L.: An Improved Clustering-based Approach for DNA Microarray Image Segmentation. In: Campilho, A.C., Kamel, M.S. (eds.) ICIAR 2004. LNCS, vol. 3212, pp. 644-652. Springer, Heidelberg (2004)

24. Callow, M.J., Dudoit, S., Gong, E.L., Speed, T.P., Rubin, E.M.: Microarray Expression Profiling Identifies Genes with Altered Expression in HDL Deficient Mice. Genome Research 10(12), 2022-2029 (2000)

25. Brown, P., Botstein, D.: Exploring the new world of the genome with DNA microarrays. Nature Genetics, 33-37 (1999)

26. Qin, L., Rueda, L., Ali, A., Ngom, A.: Spot Detection and Image Segmentation in DNA Microarray Data. Applied Bioinformatics 4(1), 1-11 (2005) 